\title{
uPAR/Cathepsin B Overexpression Reverse Angiogenesis by Rescuing FAK Phosphorylation in uPAR/Cathepsin B Down Regulated Meningioma
}

\author{
Reshu Gupta ${ }^{1}$, Arun Kumar Nalla ${ }^{1}$, Venkateswara Rao Gogineni ${ }^{1}$, Chandramu Chetty ${ }^{1}$, Praveen \\ Bhoopathi ${ }^{1}$, Jeffrey D. Klopfenstein ${ }^{2}$, Andrew J. Tsung ${ }^{2}$, Sanjeeva Mohanam ${ }^{1}$, Jasti S. Rao ${ }^{1,2 *}$
}

1 Department of Cancer Biology and Pharmacology, University of Illinois College of Medicine at Peoria, Peoria, Illinois, United States of America, 2 Department of Neurosurgery, University of Illinois College of Medicine at Peoria, Peoria, Illinois, United States of America

\begin{abstract}
Background: Meningiomas are the most commonly occurring intracranial tumors and account for approximately $15-20 \%$ of central nervous system tumors. Surgery and radiation therapy is a common treatment for brain tumors, however, patients whose tumors recur after such treatments have limited therapeutic options. Earlier studies have reported important roles of UPA, UPAR and cathepsin B in tumor progression.

Methodology/Principal Findings: In the present study, we examined the therapeutic significance of RNAi-mediated simultaneous down regulation of these proteolytic networks using two bicistronic siRNA constructs, pUC (UPAR/cathepsin B) and pU2 (UPA/UPAR) either alone or in combination with radiation in two different meningioma cell lines. Transfection of meningioma cells with pUC and pU2 significantly reduced angiogenesis as compared to control treatment both in vitro and in vivo nude mice model. This effect is mediated by inhibiting angiogenic molecules (Ang-1, Ang-2 and VEGF). Expression of focal adhesion kinase (FAK) is elevated in malignant meningioma, yet the role of intrinsic FAK activity in promoting tumor progression remains undefined. We found that pUC treatment reduced FAK phosphorylation at Y925 more efficiently compared to pU2 treatment. In immunoprecipitation assay, we found pronounced reduction of FAK (Y925) interaction with Grb2 in meningioma cells transfected with pUC with and without irradiation. Transient over-expression of uPAR and cathepsin B by full length uPAR/cathepsin B (FLpU/C) in pUC transfected meningioma cells promoted vascular phenotype, rescued expression of Ang-1, Ang-2, VEGF, FAK (Y925) and Grb2 both in vitro and in vivo mice model.

Conclusion/Significance: These studies provide the first direct proof that bicistronic siRNA construct for uPAR and cathepsin B (pUC) reduces Y925-FAK activity and this inhibition is rescued by overexpression of both uPAR and cathepsin B which clearly demonstrates that pUC could thus be a potential therapeutic approach as an anti-angiogenic agent in meningioma.
\end{abstract}

Citation: Gupta R, Nalla AK, Gogineni VR, Chetty C, Bhoopathi P, et al. (2011) UPAR/Cathepsin B Overexpression Reverse Angiogenesis by Rescuing FAK Phosphorylation in uPAR/Cathepsin B Down Regulated Meningioma. PLoS ONE 6(2): e17123. doi:10.1371/journal.pone.0017123

Editor: Irina Lebedeva, Enzo Life Biosciences, United States of America

Received October 7, 2010; Accepted January 21, 2011; Published February 11, 2011

Copyright: (C) 2011 Gupta et al. Except for The IOMM-Lee, Ang-2(70) panel in Figure 5A, the in-vivo, -IR, Ang-1 panel in Figure 5A, and the in-vivo, $+\mathrm{IR}$, MEK1 panel in Figure 6A, this is an open-access article distributed under the terms of the Creative Commons Attribution License, which permits unrestricted use, distribution, and reproduction in any medium, provided the original author and source are credited.

Funding: This research was supported by a grant from the National Institute of Neurological Disorders and Stroke (NINDS), NS61835 (to J.S.R.). The funders had no role in study design, data collection and analysis, decision to publish, or preparation of the manuscript.

Competing Interests: The authors have declared that no competing interests exist.

*E-mail: jsrao@uic.edu

\section{Introduction}

Meningiomas are the second most common primary tumor of the central nervous system, arising from the arachnoidal "cap" cells in the meninges. They constitute $\sim 20 \%$ of all intracranial primary brain tumors and are more frequent in females [1]. Most meningiomas are effectively addressed by surgical resection and radiation therapy, which is a common treatment for brain tumors. Ionizing radiation can elicit an activated phenotype that promotes rapid and persistent remodeling of the extracellular matrix (ECM) through the induction of proteases such as uPA, uPAR, and cathepsin B which suggests that inhibition of these molecules could be a potential therapeutic approach to improve the efficacy of radiotherapy $[2,3]$.

Expression of proteolytic parameters of the urokinase-type plasminogen activator system ( $\mathrm{uPA}, \mathrm{uPAR}$ ), and cathepsin $\mathrm{B}$ have been proven to be an independent prognostic parameter in cancer. These proteolytic cascades, once modified, assist several aspects of tumorigenesis. It has been shown that the content of some tumor-associated proteolytic factors in tumor extracts have a strong prognostic value. Previous studies performed by our group have established uPA, uPAR and cathepsin $\mathrm{B}$ as potential targets for therapeutic treatment of glioblastoma [4,5]. These proteolytic pathways activate a network of interconnected cell-signaling pathways that regulate cell proliferation, survival, migration and angiogenesis [6-9]. Several earlier studies have revealed that these proteolytic networks play affirmative roles in various aspects of tumorigenesis [9]. Our investigation uses this approach to examine the therapeutic significance of RNAi-mediated simultaneous down regulation of these proteolytic networks in combination with irradiation in malignant meningiomas.

As a result of UPA, uPAR and cathepsin B, interactions with certain proteins may activate intracellular signaling molecules such as focal adhesion kinase (FAK). Focal adhesion kinase (FAK) is an 
intracellular protein-tyrosine kinase, the activity of which is regulated by integrin-mediated cell adhesion [10]. In normal cells, FAK functions as an important regulator of cell motility, proliferation and anchorage-dependent cell survival [11,12]. In many tumor cells and malignant tissue, FAK expression is elevated [13] and the role of FAK in promoting tumor progression remains an area of active investigation [14]. Analyses of breast cancer tumor samples have revealed that elevated FAK expression is correlated with the development of benign ductal hyperplasia into invasive carcinomas $[15,16]$. Src-mediated phosphorylation of FAK at Y576/Y577 enhances FAK catalytic activity and Src phosphorylation of FAK at Y925 promotes Grb2 SH2-mediated binding [12,17]. However, Y925 FAK phosphorylation and Grb2 binding to FAK remain unproven as a bona fide linkage promoting mitogen-activated protein kinase (MAPK) activation [18-21]. Moreover, a role for intrinsic FAK activity or Y925 FAK tyrosine phosphorylation in tumorigenesis remains unknown.

Here we showed that si-RNA-mediated simultaneous down regulation of $\mathrm{uPA}, \mathrm{uPAR}$ and cathepsin $\mathrm{B}$ either alone or in combination with irradiation forms avascular tumors by significantly inhibiting Ang-1, Ang-2, VEGF and Y925-FAK expression both in vitro and in vivo as compared to $\mathrm{pSV}$; however, $\mathrm{pUC}$ showed relatively more pronounced effect over pU2. Therefore, we continued our study with pUC. We also discovered that over expression of uPAR and cathepsin B by FL-uPAR/cathepsin B results in rescued expression of Ang-1 Ang-2, VEGF, FAK (Y925) and Grb2 in pUC-transfected meningioma cells. Therefore, our results are the first to show that pUC inhibits intrinsic FAK (Y925) activity and Grb2 expression and thus reduces tumor angiogenesis and this effect is reversed by over expression of UPAR and cathepsin B. Our study suggests that inhibition of uPAR/cathepsin B by pUC could be a potential therapeutic approach by targeting FAK phosphorylation and can be used as anti-tumor agents in meningioma.

\section{Results}

Effect of ionizing radiation alone and in combination with pUC and pU2 on angiogenesis in meningioma cell lines

To assess the effect of ionizing radiation on angiogenesis, both meningioma cell lines were irradiated at a dose of 5 and 10gy, and in vitro capillary-like structure formation assay was performed with human microvascular endothelial cells to examine the importance of tumor-conditioned medium in the angiogenic process. We observed that $5 \mathrm{gy}$ of irradiation induced angiogenesis in IOMM-Lee when compared with control $(1.5$ fold; $\mathrm{p}<0.05)$ and 10 gy irradiation $(1.3$ fold) made them radio resistant; however, reduced angiogenesis was shown by the $\mathrm{CH}-157 \mathrm{MN}$ cells conditioned medium at both the $5 \mathrm{gy}$ $(30 \%)$ and $10 \mathrm{gy}(40 \%)$ dose in human microvascular endothelial cells (Fig. 1A, B). We wanted to observe the effect of treatment on radioresistant and radiosensitive cells and elected to conduct further experiments with a radiation dose of $5 \mathrm{gy}$ for IOMM-Lee and $10 \mathrm{gy}$ for the CH-157MN cell line. To further test the role of uPA, uPAR and cathepsin $\mathrm{B}$ we silenced these genes by si-RNA-mediated pUC (uPAR/cathepsin B) and pU2 (uPA/uPAR) constructs. We found a significant decrease of UPAR in IOMM-Lee, CH-157MN and in vivo samples when transfected with pUG and pU2 either alone or in combination with irradiation (Fig. 1G). To determine the effect of $\mathrm{pUC} / \mathrm{pU} 2$ bicistronic constructs on angiogenesis of conditioned medium from meningioma cells, we performed vessel formation assay in human microvascular endothelial cells (Fig. 1D). Results showed that si-RNA-mediated bicistronic constructs of UPAR and cathepsin $\mathrm{B}$ (pUC) efficiently reduced angiogenesis compared to pU2 (IOMMLee: $37.5 \%$; CH-157MN: $50 \%$ ) in both cells. We further studied the combined effect of these bicistronic constructs and irradiation on the angiogenesis of cells, and found that combined treatment of pUC+IR reduced vessel formation in both cell lines when compared with pSV+IR (IOMM Lee: 75\%; CH-157MN: 85\%; p<0.05), and pU2+IR (IOMM Lee: 33\%; CH-157MN: 55\%) (Fig. 1E). Results are expressed as mean $\pm \mathrm{SD}$ of three independent experiments. These results also show that angiogenesis enhanced in endothelial cells by irradiation in conditioned medium of IOMM-Lee cells was again being suppressed by $\mathrm{pUC}+\mathrm{IR}$ and $\mathrm{pU} 2+\mathrm{IR}$; however $\mathrm{pUC}+\mathrm{IR}$ was found to be relatively more pronounced.

RNAi-mediated simultaneous down regulation of UPAR and cathepsin B significantly reduces the expression of angiogenic molecules compared to $\mathrm{pU} 2$ both in vitro and in vivo

Because the alteration in Ang-1, Ang-2, and VEGF is known to promote angiogenesis, we evaluated the involvement of these molecules in transfected cells. Our results indicates that simultaneous down regulation of $\mathrm{uPAR}$, and cathepsin $\mathrm{B}$ reduced the expression of angiogenic molecules in IOMM-Lee and in CH157MN cells when compared with pSV (IOMM Lee: Ang-1 90\%, Ang-2 75\%, VEGF 65\%; CH-157MN: Ang-1, 90\%, Ang-2 $80 \%$, VEGF $75 \%$; $\mathbf{p}<0.05)$ and pU2 (IOMM Lee: Ang-1 $72 \%$, Ang-2 50\%, VEGF 50\%; CH-157MN: Ang-1 72\%, VEGF 50\%). The combination effect of $\mathrm{pUC}$ and irradiation was found to be more effective when compared with PSV+IR (IOMM Lee: Ang$187 \%$, Ang-2 81\%, VEGF 71\%; CH-157MN: Ang-1 76\%, Ang$288 \%$, VEGF $50 \%$ p $<0.05$ ) and $\mathbf{p U 2 + I R}$ (IOMM Lee: Ang-1 42\%, p <0.05: Ang-2 60\%, VEGF 50\%; CH-157MN: Ang-1 50\%, Ang-2 50\%, VEGF 20\%) (Fig. 2A, B). Our in vivo findings suggest that transfection either alone or in combination with irradiation reduces the vessel formation in nude mice $(\mathbf{F i g}$. $\mathbf{2 C}$ ) by reducing the expression of angiogenic molecules both at the RNA (Fig. 2D) and protein level (Fig. 2E). We discovered the enhanced expression of angiogenic molecules in irradiated control at the protein level (Ang-1: 400\%, Ang-2: 20\%, VEGF: 350\%) when compared with nonirradiated control, which is again down regulated by combination treatment of pUC +IR (Ang-1 70\%: Ang-2 58\%: VEGF 51\%). We also discovered the pronounced effect of pUC +IR over pU2+IR in downregulation of Ang-1 $(25 \%)$, while we detected no significant difference in the level of Ang-2 and VEGF. Results are expressed as mean \pm SD. These results show that in vivo enhanced expression of angiogenic molecules by irradiation in $\mathrm{pSV}$ has been suppressed by both pUC+IR and pU2+ IR, however pUC + IR was found to be relatively more effective $(\mathbf{F i g} . \mathbf{2 F})$.

\section{Simultaneous downregulation of UPA, UPAR and cathepsin-B reduce expression of p-FAK (Y925) and} inhibit its interaction with Grb2 both in vitro and in vivo

Many angiogenesis related genes are transcriptionally regulated by FAK. FAK mediates angiogenesis through a linear pathway involving Grb-2, H Ras and MEK1 activation. Because FAK is a major modulator of angiogenesis, we examined the status of FAK in transfected meningioma cells along with irradiation. For this, we conducted western analysis and observed significant increase $(600 \% ; \mathrm{p}<0.05)$ in $\mathrm{Y} 925$ phosphorylation of FAK in irradiated IOMM-Lee cells compared to nonirradiated cells. We also found that treatment of cells with $\mathrm{pUC}$ leads to reduction in the p-FAK (925) expression when compared with pSV (88\%) and pU2 (30\%). In contrast to IOMM-Lee cells, CH-157MN cells show reduced Y925 FAK expression in irradiated cells compared to nonirradiated cells $(65 \%)$. Our in vivo studies revealed increased 


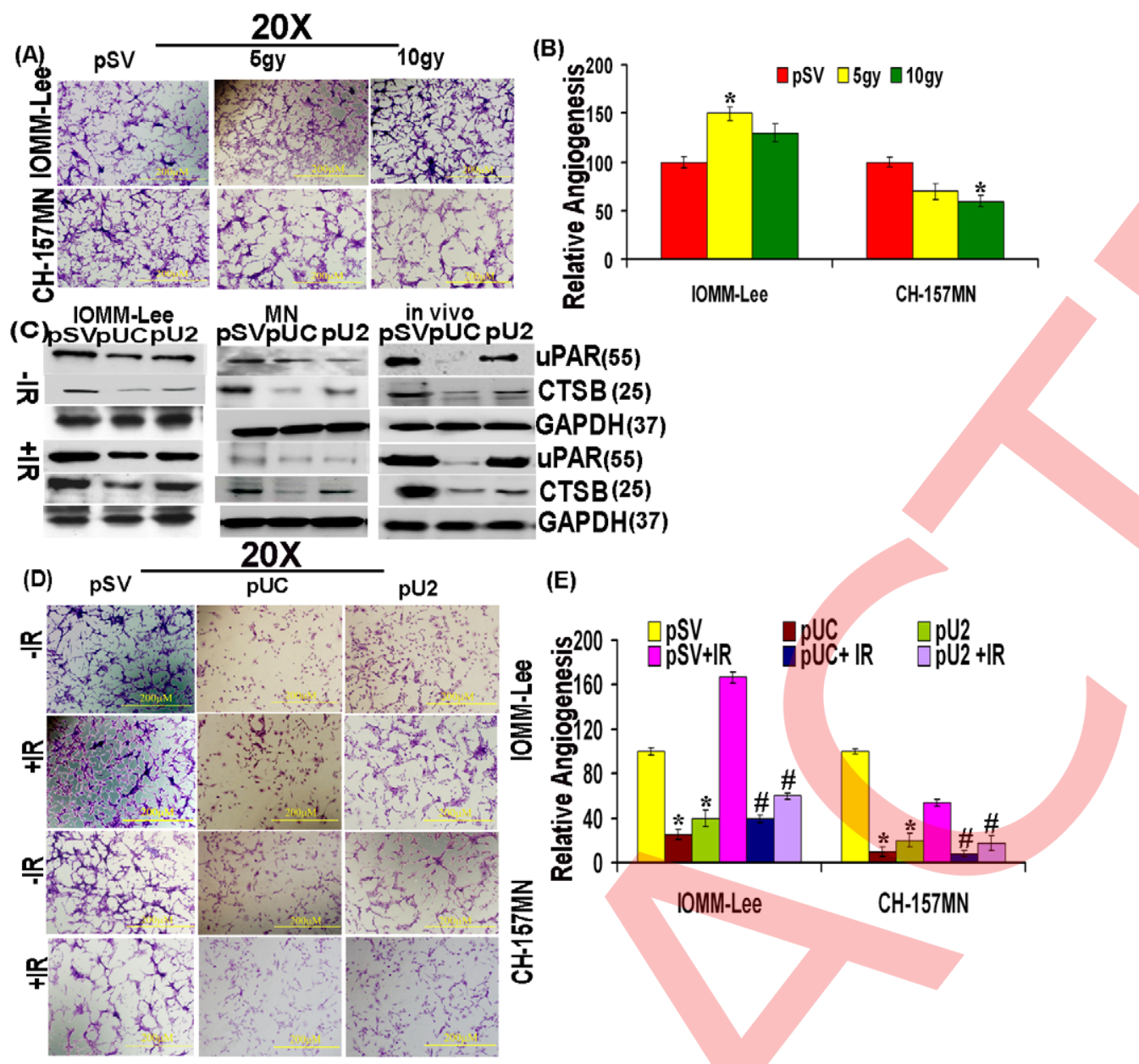

Figure 1. Effect of ionizing irradiation either alone or in combination with pUC and pU2 in human meningioma cell lines. (A, B) Conditioned medium of two meningioma cell lines, IOMM-Lee and CH-157MN, was collected after giving 5 and 10 gy doses of $\gamma$-rays irradiation. Human microvascular endothelial cells were seeded in 96-well plates and cultured with conditioned medium of meningioma cells. The cells were maintained for $48 \mathrm{hrs}$ at $21 \% \mathrm{O}_{2}$, washed, fixed, stained with Hema-3, and photographed. The percentages of angiogenic cells were assessed using Cell Quest software. Columns represent means \pm SD of three independent experiments. ${ }^{*} p<0.05$, significant difference from $p S V(C) 1 \times 10^{5}$ IOMMLee and CH-157MN cells were transfected with pSV, pUC and pU2 and cell lysates were subjected to SDS-PAGE, and immunoblot analysis was carried out to detect uPAR level. Similarly, tissue lysates isolated from nude mice were also subjected for western blotting to detect uPAR expression. (D) Human microvascular endothelial cells were seeded in 48-well plates and cultured with conditioned medium collected from pSV, pUC and pU2 transfected IOMM-Lee and $\mathrm{CH}-157 \mathrm{MN}$ meningioma cells. The cells were kept in a humidified atmosphere containing $5 \% \mathrm{CO} 2$ at $37^{\circ} \mathrm{C}$ for $48 \mathrm{~h}$, washed, fixed and stained with Hema-3 and photographed. (E) Columns represent means \pm SD of three independent experiments. ${ }^{*}$ and \# denotes $p<0.05$, significant difference from $\mathrm{pSV}$ and $\mathrm{pSV}+\mathrm{IR}$, respectively.

doi:10.1371/journal.pone.0017123.g001

expression of p-FAK in irradiated control as compared to nonirradiated control treated mice $(600 \% ; \mathrm{p}<0.05)$. The activated form of $\mathrm{pFAK}$ was down regulated by $\mathrm{pUC}$ in combination with irradiation when compared with pSV+IR $(96 \% ; \mathrm{p}<0.05)$ and $\mathrm{pU} 2$ $+\operatorname{IR}(54 \%)$ in nude mice $($ Fig. 3A, B). In vivo immunofluorescence studies in paraffin embedded tumor sections showed the same results (Fig. 3C). To test the significance of decreased pY925 phosphorylation and to determine whether FAK forms a signaling complex with Grb2 in meningioma cells, co-immunoprecipitation analyses were performed. Since pUC caused more reduction of Y925, angiogenic molecules and angiogenesis when compared with pU2, we continued our study with pUC only. The Grb2 association with FAK was inhibited in pUC-transfected meningioma IOMMLee $(20 \%)$, CH-157MN (70\%) and in tumor sections of nude mice $(75 \%)$ when compared with pSV. The combination treatment of pUC + IR again reduced interaction of p-FAK with Grb2 when compared with pSV+IR in IOMM-Lee (55\%), CH-157MN (40\%) and in nude mice $(90 \%)$ (Fig. 3D, E). As Grb2 binding to FAK is a possible link to extracellular signal-regulated kinase, MAP kinase and $\mathrm{H}$ Ras activation, phospho-specific antibodies were used to evaluate mitogen-activated protein kinase kinase 1 (MEK1) and $\mathrm{H}$ Ras in lysates of pUC transfected meningioma cells. These results are consistent with the loss of signaling through a FAK-Grb2-RasMEK1 pathway upon simultaneous inhibition of uPAR/cathepsin $\mathrm{B}$ in meningioma cells (Fig. 3).

uPAR/cathepsin B over expression rescues angiogenic defect of pUG-transfected meningioma both in vitro and $i n$ vivo. To prove that the inhibition of $U P A R$ and cathepsin $B$ leads to reduced vessel formation, uPAR and cathepsin B were over-expressed in pUC-transfected cells via co-transfection with FL-uPAR and FL-cathepsin B. We found rescued uPAR and cathepsin B (Fig. 4A) expression in nonirradiated pUC-transfected cells (IOMM-Lee: uPAR 1.8 fold, cathepsin B 1.78 fold; CH157MN: uPAR 4.5 fold, cathepsin B 4.75 fold; nude mice: uPAR 


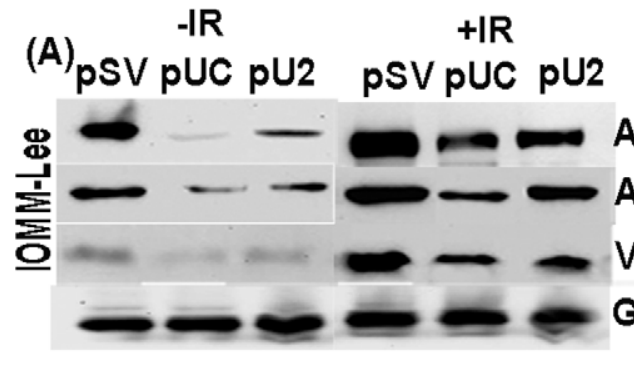

(B)

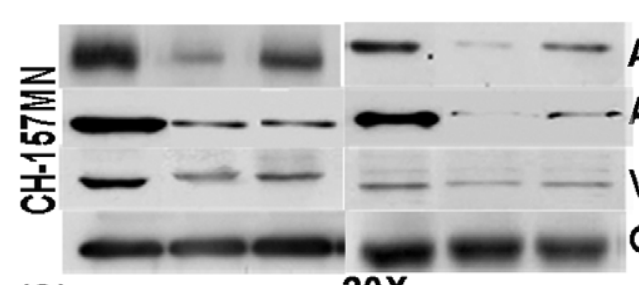

(C)
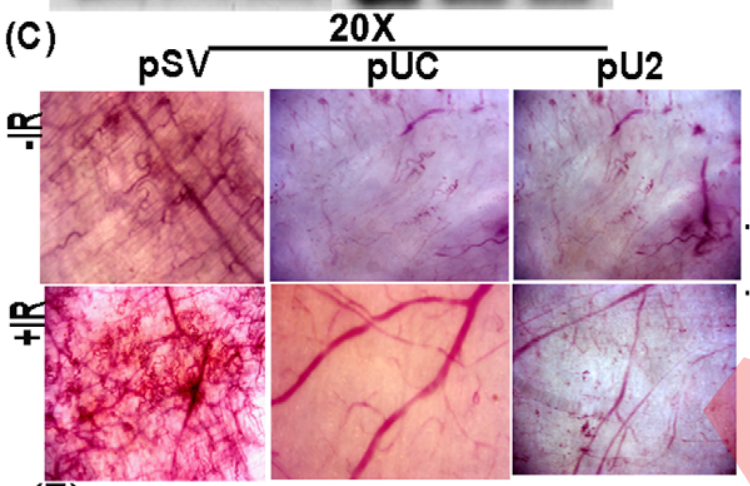

(E)

-IR

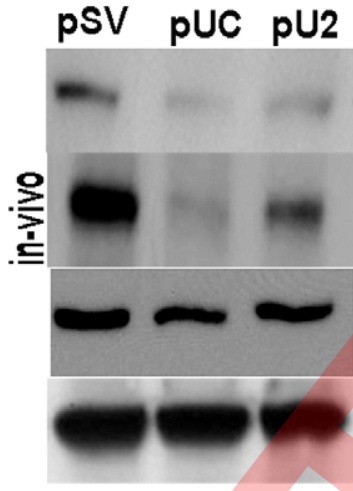

+ IR

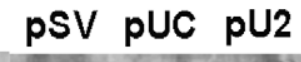

(F)

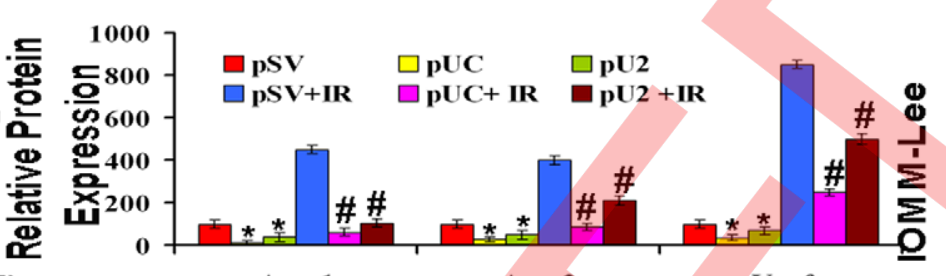

Ang-1

Ang-2

Vegf GAPDH(37)

VEGF(15) $\frac{\text { के }}{\mathbf{m}}$

GAPDH(37)
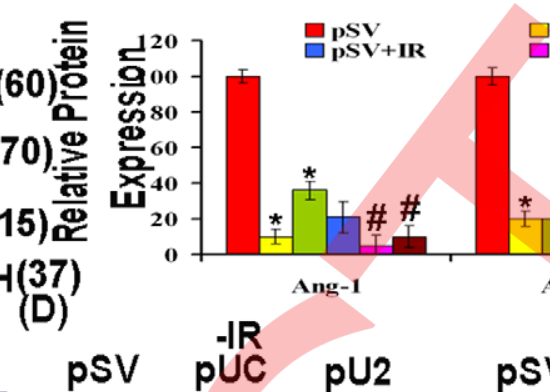

品UC

$\square_{\mathrm{pU} 2}^{\mathrm{pU}+\mathrm{IR}}$
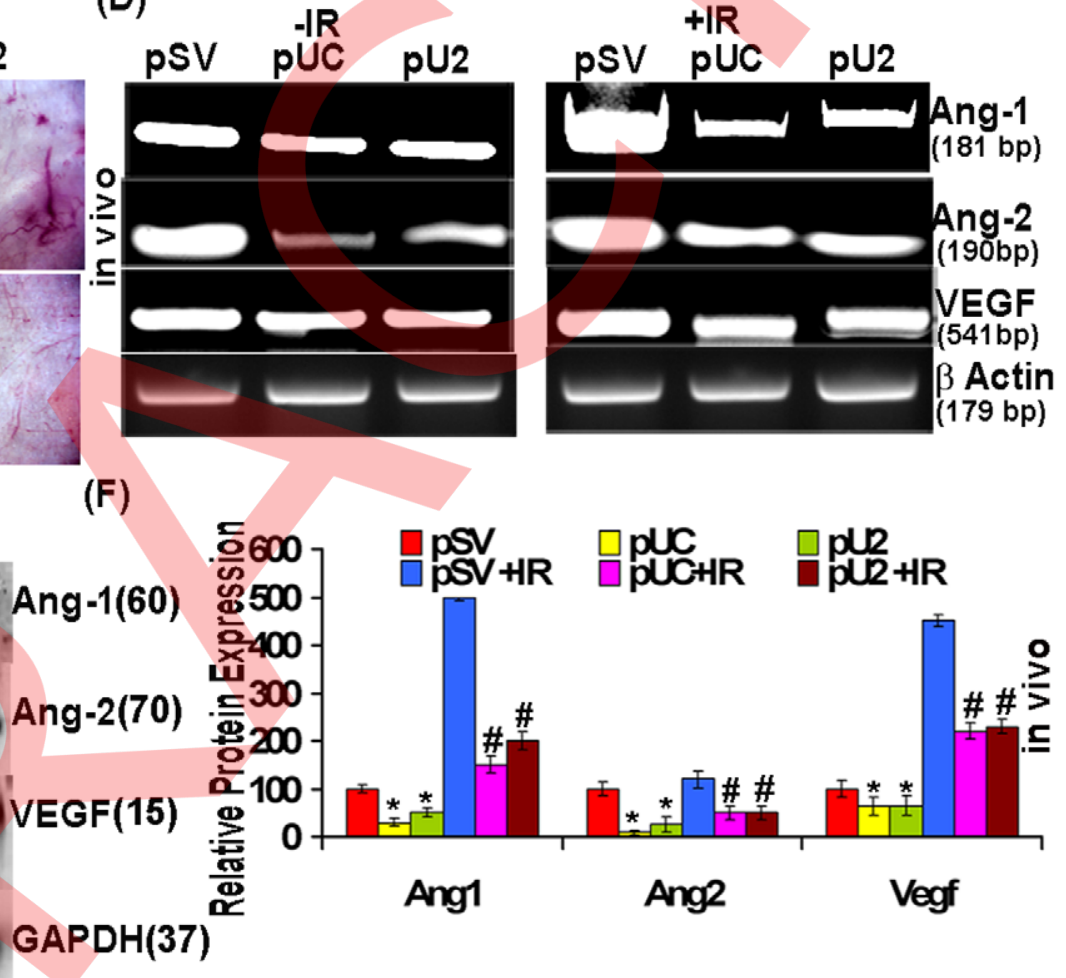

Figure 2. Simultaneous down regulation of UPA, UPAR and cathepsin B inhibits expression of angiogenic molecules in meningiomas both in vitro and in vivo. (A) IOMM-Lee and CH-157MN cells were transfected with pSV, pUC and pU2 and irradiated as described earlier. Equal amounts of protein from control and treated cell lysates were analyzed by western blotting using specific primary antibodies for Ang-1, Ang-2, and VEGF. GAPDH served as a loading control. (B) Band intensities were quantified by densitometry. Columns represent means \pm SD of three independent experiments. * and \# denotes $p<0.05$, significant difference from pSV and pSV+IR, respectively (C) Athymic nude mice ( $\mathrm{n}=48, \mathrm{nu} / \mathrm{nu}, 8$ mice for each group) were bred and maintained within a specific pathogen-free environment. Transfected IOMM-Lee cells were implanted with dorsal skin-fold chamber preparation that contained 2 layers of the membrane on both sides of a ring. This model is characterized by both solid and diffuse infiltrative growth patterns. Orthotopic tumors were established through intracranial injection of IOMM-Lee cells into brains of athymic nude mice. Once the control group showed chronic symptoms, the animals were euthanized by cardiac perfusion. RT-PCR (D) and western blotting (E) was performed on nonirradiated and irradiated control and treated tissue lysates by using specific primers/antibodies (Ang-1, Ang-2, VEGF) for angiogenic proteins. (F) Band intensities were quantified by densitometry. Columns represent means \pm SD. ${ }^{*}$ and \# denotes $p<0.05$, significant difference from pSV and pSV+IR, respectively.

doi:10.1371/journal.pone.0017123.g002

2.25 fold, cathepsin B 1.78 fold), and irradiated pUC-transfected cells (IOMM-Lee: uPAR 3 fold, cathepsin B 3.2 fold; CH-157MN: uPAR 2 fold, cathepsin B 2.6 fold; nude mice: uPAR 4.8 fold, cathepsin B 1.65 fold) after co-transfection with FL-uPAR and FLcathepsin B (Fig. 4B). Both in vivo and in vitro findings suggest that co-transfection with FL-uPAR and FL-cathepsin B rescues the vessel formation in pUC-transfected cells (Fig. 4C).

uPAR/cathepsin over expression rescues expression of angiogenic molecules in pUC-transfected meningioma both in vitro and in vivo. We observed that co-transfection with FL- 


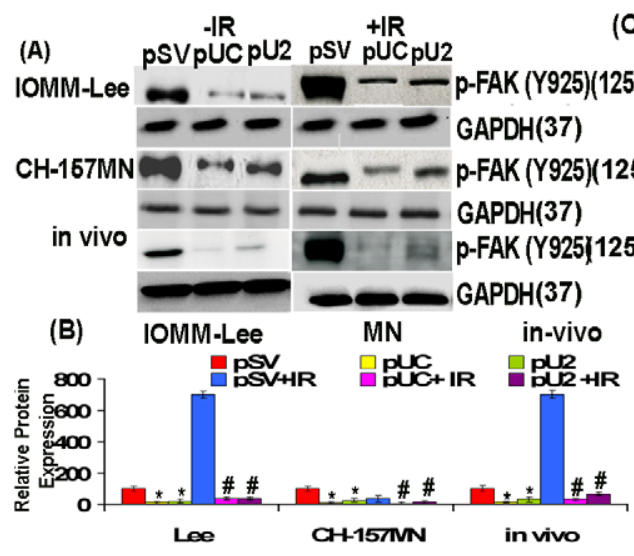

IP: p-FAK(Y925)

(D)pSV pUC pSV+IR pUC+IIR (E)
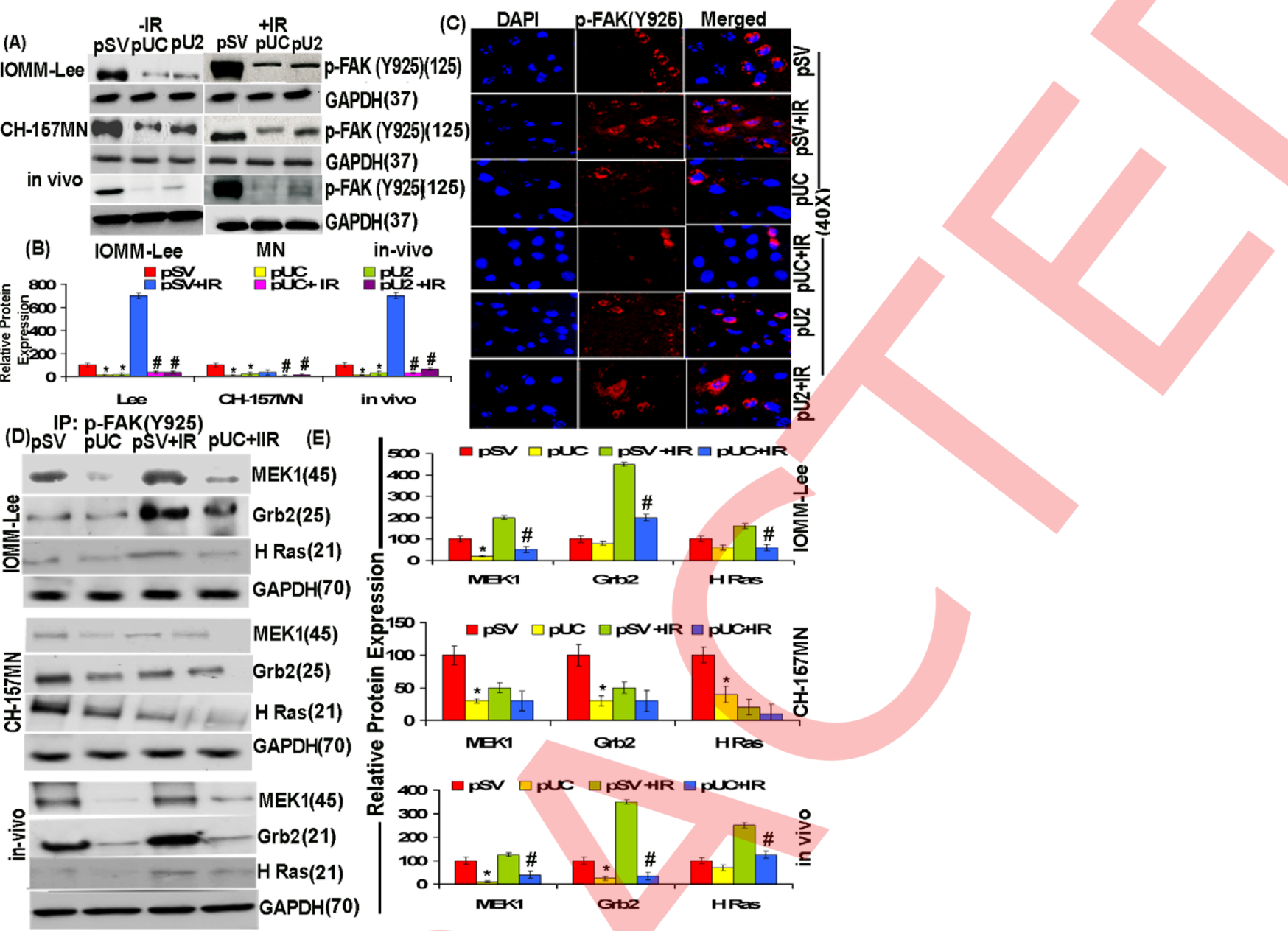

Figure 3. Y925 phosphorylation of FAK and its interaction with Grb2 is suppressed by simultaneous down regulation of uPA, uPAR and cathepsin B either alone or in combination with irradiation both in vitro and in vivo. (A) Western blotting was performed on nonirradiated and irradiated control and treated tissue lysates by using specific p-FAK (925) antibody. (B) Band intensities were quantified by densitometry. Columns represent means \pm SD of three independent experiments. * and \# denotes $p<0.05$, significant difference from pSV and pSV+IR, respectively. (C) Immunofluorescence analysis was performed for p-FAK expression in paraffin-embedded tumor sections. Fields with intense fluorescence were scored for protein expression. (D) To test the significance of decreased pY925 phosphorylation and to determine whether FAK forms a signaling complex with Grb2 in meningioma cells, co-immunoprecipitation analyses were performed by using antibodies for MEK1, Grb-2, and $\mathrm{H}$ Ras both in vitro and in vivo. (E) Band intensities were quantified by densitometry. Columns represent means \pm SD of three independent experiments. * and \# denotes $p<0.05$, significant difference from pSV and pSV+IR, respectively.

doi:10.1371/journal.pone.0017123.g003

uPAR and FL-cathepsin $\mathrm{B}$ rescues the expression of angiogenic molecules in pUC-transfected IOMM-Lee cells (Ang-1 4.0 fold, Ang-2 2.0 fold, VEGF 2.0 fold), CH-157MN cells (Ang-1 1.8 fold, Ang-2 2.8 fold, VEGF 4.16 fold) and in nude mice (Ang-1 1.4 fold, Ang-2 2.5 fold, VEGF 2.1 fold) (Fig. 5A, B). We also found that FL-uPAR and FL-cathepsin B rescues the expression of angiogenic molecules in pUG +IR transfected cells (IOMM-Lee: Ang-1 2.5 fold, Ang-2 3.2 fold, VEGF 2.0 fold; CH-157MN: Ang-1 1.6 fold, Ang-2 1.5 fold, VEGF 2.4 fold; nude mice: Ang-1 2.5 fold, Ang21.34 fold, VEGF 1.8 fold) (Fig. 5A, B). This data shows that over-expression of $\mathrm{UPAR}$ and cathepsin $\mathrm{B}$ might enhance angiogenesis in pUC-transfected cells by reversing the inhibitory effect of pUC on angiogenic molecules.

uPAR/cathepsin B overexpression rescues p-FAK (925) both in vitro and in vivo

Over expression of uPAR and cathepsin B by FL-uPAR and FL-cathepsin B rescued p-FAK (925), MEK1 and Grb2 expression in pUC-transfected IOMM-Lee (p-FAK 3.0 fold, MEK1 2.0 fold, Grb2 6.0 fold), CH-157MN (p-FAK 3 fold, MEK1 3 fold, Grb2 4 fold; $\mathrm{p}<0.05)$ and in nude mice ( $\mathrm{p}-\mathrm{FAK} 1.5$ fold, MEK1 1.4 fold, Grb2 3 fold). Similarly, it also rescued expression of p-FAK (925), MEK1 and Grb2 expression in pUC+IR transfected IOMM-Lee cells(p-FAK 2 fold, MEK1 2.2 fold, Grb2 2.5 fold; p $<0.05)$, CH$157 \mathrm{MN}$ (p-FAK 2 fold, MEK1 1.6 fold, Grb2 1.5 fold; p<0.05) and in nude mice (p-FAK 2.5 fold, MEK1 1.5 fold, Grb2 5 fold; $\mathrm{p}<0.05)$ (Fig. 6A, B). These results suggest that simultaneous down regulation of $\mathrm{UPAR}$ and cathepsin $\mathrm{B}$ either alone or in combination with irradiation form avascular tumors by down regulating FAK(Y925) phosphorylation, which is reversed by the over expression of UPAR and cathepsin B and thus may facilitate an angiogenic switch in tumors.

\section{Discussion}

The over expression of uPA, uPAR and cathepsin B by radiation has been detected in various malignancies, including 

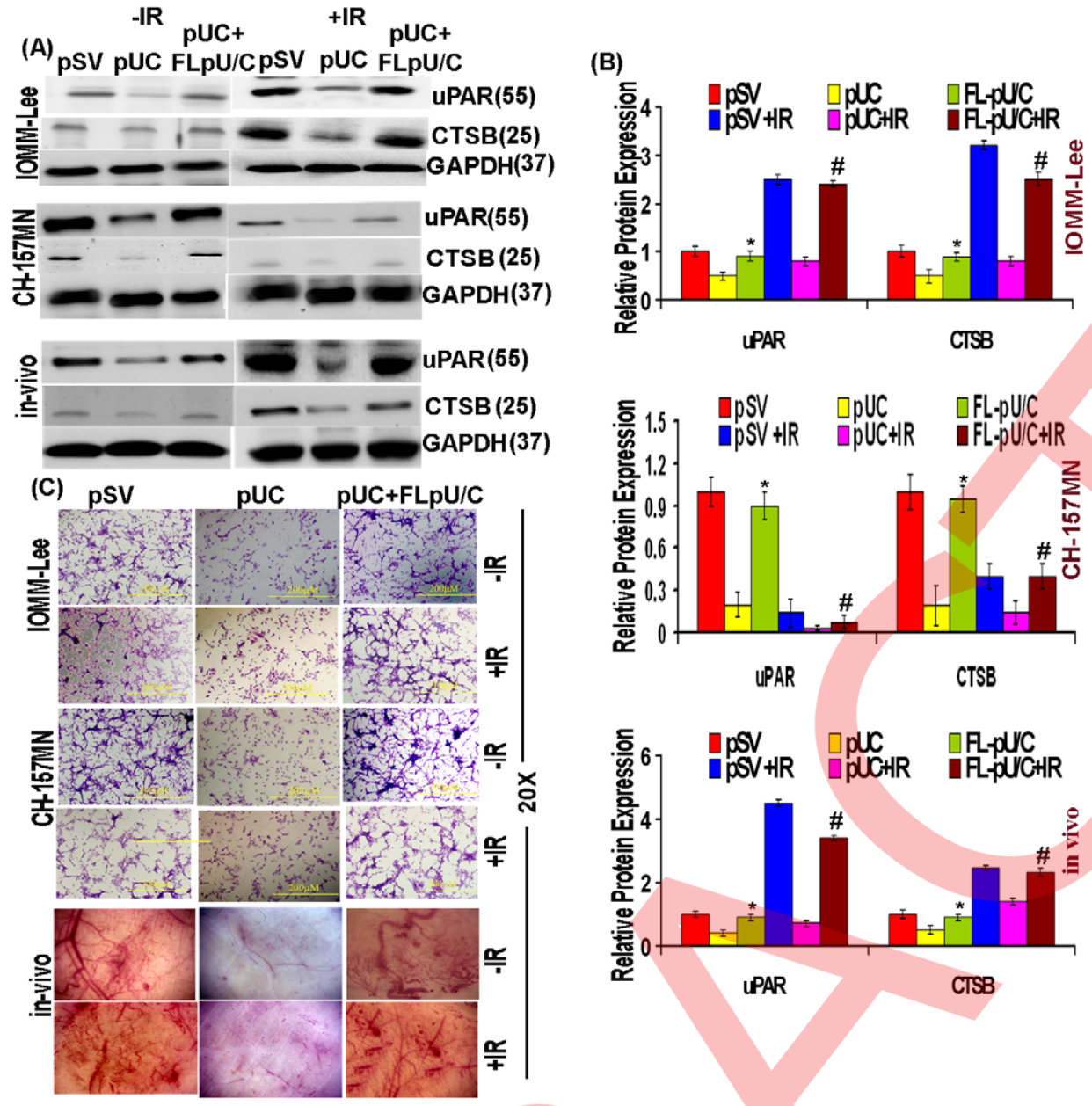

Figure 4. Over expression of UPAR and cathepsin B rescues vascular phenotype in meningiomas both in vitro and in vivo. (A) Nonirradiated and irradiated control and treated tissue lysates collected after co- transfection with FL-uPAR/FL-cathepsin B (FLpU/C) were subjected to SDS-PAGE. Immunoblot analysis was carried out to detect UPAR and cathepsin level. (B) Band intensities were quantified by densitometry. Columns represent means \pm SD of three independent experiments. ${ }^{*}$ and \# denotes $p<0.05$, significant difference from pUC and pUC+IR, respectively. (C) Vessel formation assay was performed in FL-UPAR/FL-cathepsin B (FLpU/C) transfected IOMM-Lee and CH-157MN cells and in nude mice.

doi:10.1371/journal.pone.0017123.g004

those in hepatocellular [22], rectal [23] and breast [24] cancer. Most of the cellular responses modulated by these proteolytic networks, including migration, cellular adhesion, differentiation, proliferation and angiogenesis require transmembrane signaling, which is mediated by direct contacts of these proteolytic networks with a variety of extracellular proteins and membrane receptors. In the literature it has been provided that UPA-uPAR binding results in the expression of cathepsin B and MMP-9 in monocytic cells [25] as well as cathepsin B indirectly activates other enzymes including soluble and receptor bound uPA [26].

As a preliminary assessment of the potential of RNAi-mediated two bicistronic constructs $\mathrm{uPAR} /$ cathepsin $\mathrm{B}(\mathrm{pUC})$ and $\mathrm{uPA} /$ uPAR (pU2) as a therapeutic agent in this setting, we assessed their in vitro and in vivo angiogenic activity against primary meningioma cells. When transfected with bicistronic constructs, meningioma cells form an avascular tumor phenotype. This avascular phenotype was mediated by inhibition of angiogenic molecules. Among two bicistronic constructs, pUG was found to be the most effective mediator of angiogenesis both in vitro and in vivo as confirmed by vessel formation assay when compared with pU2 and pSV. However, the combined treatment of pUG with irradiation was found to be relatively more effective over pU2+IR and irradiated control both in vitro and in vivo. We also observed that $\mathrm{pUC}$ and pU2 treated cells showed reductions in cell proliferation after radiation as compared to the controls and irradiated control as confirmed by BrdU assay (Fig. S1B). Despite the increase in angiogenesis, we found reduce cellular proliferation in irradiated control when compared with nonirradiated control. The reduction in cell proliferation in irradiated cells versus nonirradiated cells may be due to DNA damage caused by radiation, which might enhance delay in progression of the cell cycle. It has been shown that angiogenesis has inverse correlation with proliferation while at the same time cellular proliferation has positive correlation with apoptosis in invasive ductal carcinoma of breast [27].

Moreover, over expression of uPA, uPAR, or cathepsin B by tumor cells has been shown $[28,29]$ as has the potent angiogenic activity of these proteolytic networks in epithelial cells which prompted us to test the possibility that these proteolytic networks might regulate FAK expression in meningiomas, a possibility that has not been previously explored to our knowledge. In this study we have shown that si-RNA-mediated simultaneous down 

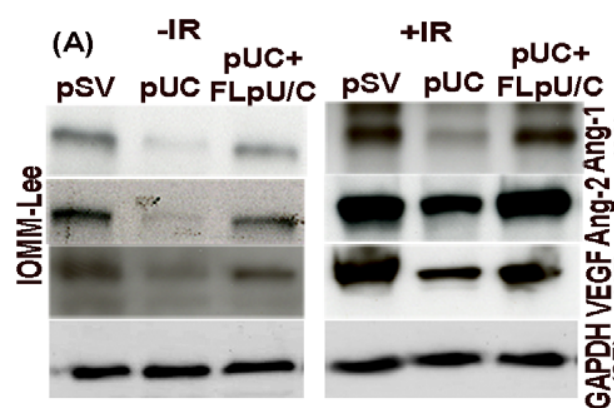

(B)
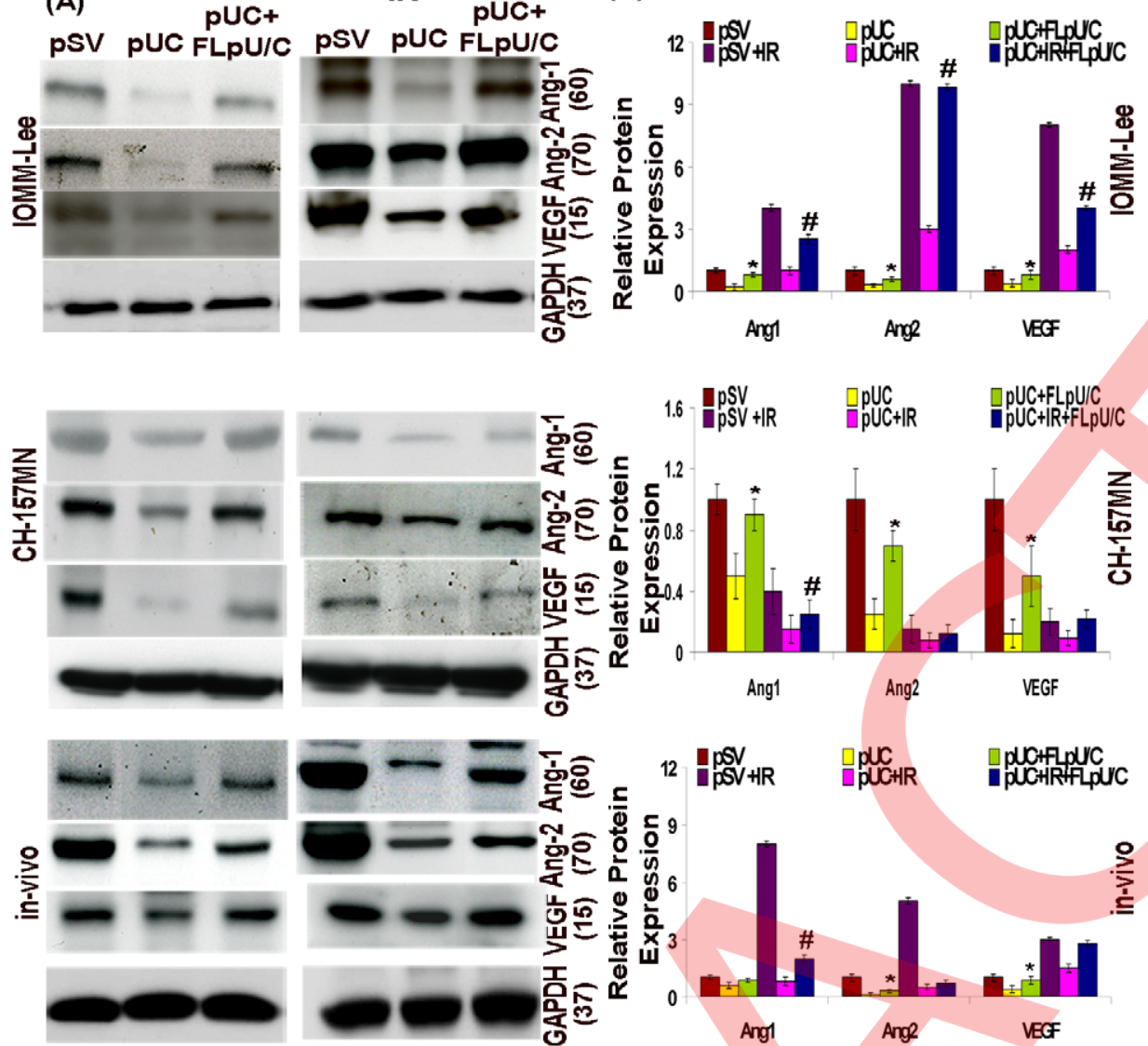

Ang1

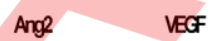

Figure 5. Over expression of uPAR and cathepsin B rescues angiogenic molecules. (A) After co-transfection with FL-uPAR/FL-cathepsin B (FLpU/C), western blotting was performed on nonirradiated and irradiated control and treated tissue lysates by using specific antibodies (Ang-1, Ang2, VEGF) for angiogenic proteins. (B) Band intensities were quantified by densitometry. Columns represent means \pm SD of three independent experiments. ${ }^{*}$ and \# denotes $p<0.05$, significant difference from pUC and pUC+IR, respectively. The IOMM-Lee, Ang-2(70) and in-vivo, -IR, Ang-1 panels in Figure 5A are excluded from this article's CC-BY license. See the accompanying retraction notice for more information. doi:10.1371/journal.pone.0017123.g005

regulation of uPAR and cathepsin B by pUC leads to significant inhibition of Y397 (figure S1A), Y925 phosphorylation of FAK, less VEGF secretion, or thus leads to small avascular tumors both in vitro and in vivo when compared with nonirradiated controls and pU2. We also found relatively reduced angiogenesis by pUC+IR when compared with irradiated controls and pU2+IR in IOMMLee, CH-157MN meningioma cells and in nude mice. It has been shown that targeted deletion of FAK suppressed chemically induced skin tumor formation in mice [30] and reduced apoptosis was observed in both normal and tumor cells after FAK deletion [30,31]. To our knowledge this is the first study showing reduced expression of p-FAK (Y925) by pUC in meningioma cells both in vitro and in vivo, which may play a role in tumor suppression by reduced expression of p-FAK (Y925). Despite correlations between elevated FAK expression and an aggressive tumor phenotype $[13,32]$, there is limited information on how FAK signaling contributes to tumor progression in situ [14]. It has been shown that FAK inhibition via stable FRNK leads to inhibition of FAKGrb2-MAPK-signaling linkage and thus also inhibits VEGF expression [33]. The biological importance of this FAK-signaling pathway was confirmed through reconstitution experiments using Src-transformed FAK-null fibroblasts where point-mutations affecting FAK catalytic activity or Y925 phosphorylation disrupted the ability of FAK to promote MAPK- and VEGF-associated tumor growth [33]. We also found that pUC inhibited a FAK-
Grb2-MEK1-Ras signaling linkage regulating VEGF expression. These results clearly show the involvement of pUC in inhibiting pFAK (Y925) signaling with or without irradiation.

Despite the strong correlations between FAK and tumor metastasis, the molecular mechanism(s) through which FAK can promote these events has yet to be established. Analyses of FAK expression levels in esophageal squamous cell carcinoma [34], hepatocellular carcinoma [35], acute myeloid leukemia [36] and ovarian cancer [37] tissue samples have found that FAK expression levels are inversely correlated with patient survival. Taken together with the fact that FAK catalytic activity promotes tumor metastasis, the above mentioned studies strongly support the therapeutic targeting of FAK in the treatment of cancer [33]. As pUC is inhibiting Y925 phosphorylation, we over-expressed uPAR and cathepsin B by FLpU/C to demonstrate the role of these molecules in relation to $\mathrm{Y} 925$ phosphorylation. The biological importance of this FAK-signaling pathway was confirmed through reconstitution experiments using FL-uPAR/ cathepsin B-transformed meningioma cells where overexpression of uPAR and cathepsin B rescued Y925 phosphorylation, MEK1, Grb2, Ang-1, Ang-2 and VEGF expression. We also found the increase in cellular proliferation in pUC transfected cells in both meningioma cell lines upon overexpression of UPAR and cathepsin by FL-uPAR/FL-cathepsin B (FLpU/C) as confirmed by BrdU assay (figure S1C). Since catalytic activity of FAK promotes tumor 


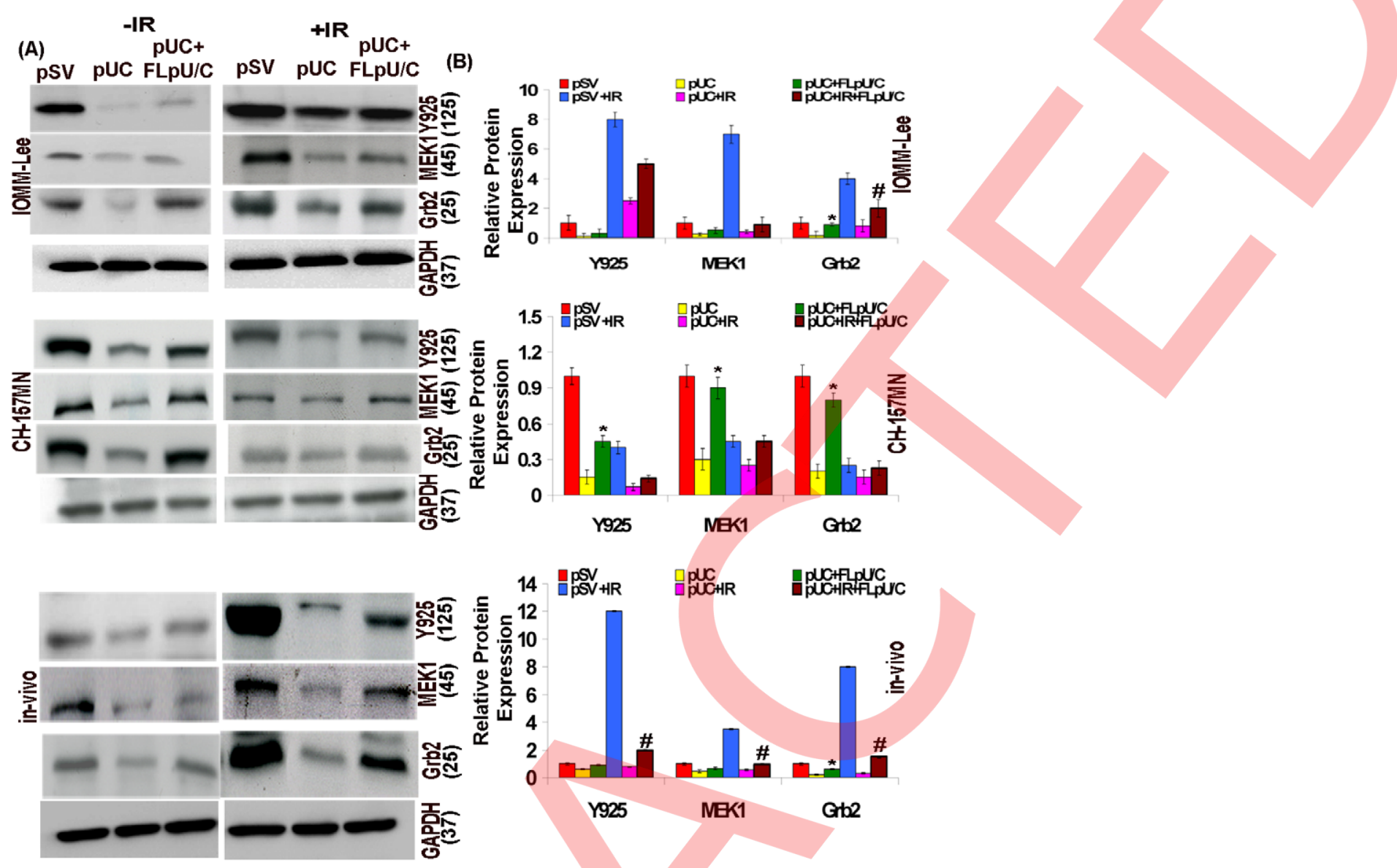

Figure 6. Over expression of UPAR and cathepsin B rescues Y925-FAK phosphorylation. (A) After co-transfection with FL-uPAR/FLcathepsin B (FLpU/C) western blotting was performed on nonirradiated and irradiated control and treated tissue lysates by using specific antibodies (Y925-FAK, MEK1 and Grb2) (B) Band intensities were quantified by densitometry. Columns represent means \pm SD of three independent experiments. *and \# denotes $p<0.05$, significant difference from pUC and pUC+IR, respectively. The in-vivo, +IR, MEK1 panel in Figure $6 \mathrm{~A}$ is excluded from this article's CC-BY license. See the accompanying retraction notice for more information. doi:10.1371/journal.pone.0017123.g006

angiogenesis, these results strongly support the therapeutic targeting of FAK by pUC in the treatment of meningioma.

\section{Materials and Methods}

\section{Ethics Statement}

The Institutional Animal Care and Use Committee of the University Of Illinois College Of Medicine at Peoria, Peoria, IL, USA, approved all surgical interventions and post-operative animal care. The consent was written and approved. The approved protocol numbers are 817 dated November 1, 2007 and renewed on May 13, 2010, and 851, dated April 23, 2009 and renewed on May 12, 2010.

\section{Construction of siRNA and FL-uPAR/cathepsin B- expressing plasmids}

Two bicistronic constructs expressing shRNA for uPAR/ cathepsin $\mathrm{B}(\mathrm{pUC})$ and $\mathrm{uPA} / \mathrm{uPAR}(\mathrm{pU} 2)$ were constructed using a pcDNA3 vector as described previously by our group [5]. All of the vectors were expressed under the control of a CMV promoter [5]. A pcDNA3-scrambled vector with an imperfect sequence, which does not form a perfect hairpin structure, was used to develop the scrambled vector for use as a control (pSV). uPAR and cathepsin B over expressing plasmids were purchased from OriGene (Rockville, MD).

\section{Cell cultures and transfection conditions and reagents}

IOMM-Lee and $\mathrm{CH}-157 \mathrm{MN}$ meningioma cells were purchased from American Type Culture Collection. Both cell lines were cultured in high glucose containing Dulbecco's Modified Eagle's Medium. Cultures were supplemented with $100 \mu \mathrm{g} / \mathrm{mL}$ streptomycin, $100 \mathrm{U} / \mathrm{mL}$ penicillin and 10\% fetal calf serum (FCS) and maintained in a humidified atmosphere containing 5\% CO2 at $37^{\circ} \mathrm{C}$. Cells were transfected with pSV, pUC, pU2, FL-uPAR/FLcathepsin B (FLpU/C) using Fugene reagent following the manufacturer's instructions. Transfected cells were maintained in serum containing media for $48 \mathrm{~h}$ and for irradiated experiments transfected cells were further irradiated for 24 hour before use in various procedures. The primary antibodies used for this study were anti-uPAR (R\&D systems, Minneapolis, CH-157MN), pFAK, Cathepsin B, Ang-1, Ang-2, VEGF, H Ras (Santa Cruz Biotechnology, Santa Cruz, CA), MEK1, Grb2, anti-GAPDH (Cell Signaling, Boston, MA) and HRP conjugated secondary antibodies (Biomed, Foster City, CA).

\section{In vitro angiogenesis assay}

Tumor conditioned medium induced microtubule network formation was determined as described previously [7]. Irradiated and non irradiated pUC and pU2 transfected cells were incubated in serum-free medium in a humidified atmosphere containing $5 \%$ $\mathrm{CO} 2$ at $37^{\circ} \mathrm{C}$ for $24 \mathrm{~h}$. Conditioned medium was collected and 
added to human microvascular endothelial cells (HMEC-1) derived from dermis and provided by Dr. Francisco J. Candal (Centers for Disease Control and Prevention, Atlanta, GA, USA), which were seeded the previous day in 48-well plates and incubated for 48 hour. The formation of the microtubule networks was examined using a phase-contrast microscope equipped with a CG camera and evaluated by Discovery Image Pro software (Fryer, Huntley, IL). All experiments were repeated at least three times. The degree of angiogenesis was measured by the following method: number of branch points and the total number of branches per point were counted, with the product indicating the degree of angiogenesis.

Results are presented as the means $\pm \mathrm{SD}$ of eight fields.

\section{Western blot analysis}

All meningioma cells were cultured in 100-mm dishes. The cells were transfected with bicistronic constructs pUC, pU2 and FLuPAR/FL-cathepsin B (FLpU/C) for $48 \mathrm{~h}$. At the same time, control cultures were maintained in $21 \% \mathrm{O}_{2}$ for 48 hours. For irradiation experiments transfected cells were irradiated for further 24 hours. Cell extracts were prepared in ice-cold RIPA buffer (50 mmol/L Tris-HCl (pH 7.4), $150 \mathrm{mmol} / \mathrm{L} \mathrm{NaCl}, 1 \%$ IGEPAL, $1 \mathrm{mmol} / \mathrm{L}$ EDTA, $0.25 \%$ sodium deoxycholate, $1 \mathrm{mmol} / \mathrm{L}$ sodium fluoride, $1 \mathrm{mmol} / \mathrm{L}$ sodium orthovanadate, $0.5 \mathrm{mmol} / \mathrm{L}$ PMSF, $10 \mu \mathrm{g} / \mathrm{mL}$ aprotinin, $10 \mu \mathrm{g} / \mathrm{mL}$ leupeptin). The protein concentration in each extract was determined by BSA assay (Sigma, St. Louis, MO). Cell extracts were subjected to SDSPAGE and proteins were transferred to nitrocellulose membranes and probed with primary antibodies that detect Ang-1, Ang-2, VEGF, p-FAK, MEK1, H Ras, uPAR, cathepsin B and Grb2. Signals were detected by using the ECL system (Amersham Bioscience). The same membranes also were probed to detect GAPDH as a loading control.

\section{Immunofluorescence analysis of protein expression in tumor sections}

Paraffin-embedded tumor sections were subjected to rehydration by passing through a series of xylene, $100 \%$ and $90 \%$ ethanol. The slides were washed in cold PBS and blocked for $30 \mathrm{~min}$ in $1 \%$ BSA prepared in PBS followed by overnight incubation in primary antibody (p-FAK-Y925) at a concentration of $1: 100$ at $4^{\circ} \mathrm{C}$. The slides were then washed three times with $1 \%$ BSA in PBS for $2 \mathrm{~min}$ per wash, and incubated for $1 \mathrm{hr}$ in appropriate fluorescence-labeled secondary antibody. The slides were kept in the dark to avoid exposure to light. Preparations were mounted on slides using DAPI (Sigma, St. Louis, MO) and fields with intense fluorescence were scored for protein expression. All microscopy studies were performed using fluorescent microscope attached to Olympus camera and set to auto.

\section{Dorsal skin-fold chamber model}

Athymic nude mice (nu/nu; 48 female, 5-7 weeks old) were divided into 8 treatment groups with 6 animals in each group and maintained within a specific pathogen and germ-free environment. The implantation technique of the dorsal skin-fold chamber model has been described previously [38]. Sterile small-animal surgical techniques were followed. Mice were anesthetized by intraperitoneal injection with ketamine $(50 \mathrm{mg} / \mathrm{kg}) /$ xylazine $(10 \mathrm{mg} / \mathrm{kg})$. Once the animal was anesthetized completely, a dorsal air sac was made in the mouse by injecting $10 \mathrm{~mL}$ of air. Diffusion chambers (Fisher, Hampton, $\mathrm{NH}$ ) were prepared by aligning $0.45-\mu \mathrm{m}$ Millipore membranes (Fisher) on both sides of the rim of the " $\mathrm{O}$ " ring (Fisher) with sealant. Once the chambers were dry (2-3 min), they were sterilized by UV radiation overnight. Membranes were wetted with $20 \mu \mathrm{L}$ of PBS. $5 \times 10^{6} \mathrm{pSV} / \mathrm{pUC} / \mathrm{pU} 2$ and FLuPAR/ cathepsin B transfected cells either alone or in combination with irradiation were suspended in $100-150 \mu \mathrm{L}$ of sterile PBS and injected into the chamber through the opening of the " $\mathrm{O}$ " ring. The opening was sealed with a small amount of bone wax. A 1.5 to $2 \mathrm{~cm}$ superficial incision was made horizontally along the edge of the dorsal air sac and the air sac was opened. With the help of forceps, the chambers were placed underneath the skin and carefully sutured. After 15 days, the animals were anesthetized with ketamine/xylazine and sacrificed by intracardial perfusion with saline $(10 \mathrm{~mL})$ followed by $10 \mathrm{~mL}$ of $10 \%$ formalin/ $0.1 \mathrm{M}$ phosphate solution. The animals were carefully skinned around the implanted chambers and the implanted chambers were removed from the subcutaneous air fascia. The skin fold covering the chambers was photographed under visible light.

\section{Reverse transcription-PCR}

Total RNA was extracted using TRIZOL reagent (Life Technologies, Carlsbad, California) according to the manufacturer's instructions. RT-PCR was done by using the reverse transcription kit (Roche Diagnostics, Indianapolis, IN). PCR products were resolved on $1.5 \%$ agarose gel and seen by ethidium bromide staining. $\beta$ actin was used as loading control. We used the following primers:

Ang-1, Forward 5'-GAAGGGAACGGAGCGTATTC-3', and reverse 5' GGGCACATTTGCACATACAG

Ang-2, forward 5'-CCACAAATGGCATCTACACG-3', and reverse 5' - CGCAGCGAATATTCTCGTGA-3';

VEGF forward 5' - ATGAACTTTCTGCTGTCTTGGGT $3^{\prime}$, and reverse 5'- TCACGGCGTCGGCTTGTCAC - $3^{\prime}$;

$\beta$ actin, forward 5'-GTCGTAGCACTGGCATTGT-3', and reverse 5' - CAGGTGTGTGGTGGTGAAGGT - 3 '.

PCR products were resolved on $1 \%$ agarose gel, visualized, and photographed under UV light. RT-PGR for $\beta$ actin was performed to normalize input RNA.

\section{Immunoprecipitation}

Meningioma cells were grown in $100 \mathrm{~mm}$ plates, transfected with $\mathrm{pSV} \& \mathrm{pUC}$ either alone or in combination with irradiation and lysed in $1 \mathrm{ml}$ of immunoprecipitation buffer for $30 \mathrm{~min}$ on ice, with occasional swirling. One $\mathrm{ml}$ of immunoprecipitation buffer lacking detergent was then added to each plate and cells were scraped. The following immunoprecipitation buffer was used: $\pm 0.5 \%$ 3-[(3-cholamidopropyl)dimethylammonio]-1-propanesulfonic acid, $150 \mathrm{mM} \mathrm{NaCl}, 50 \mathrm{mM}$ Tris-Hcl, $2 \mathrm{mM}$ EDTA, $2 \mathrm{mM}$ EGTA, $2 \mathrm{mM} \mathrm{Na} \mathrm{VO}_{4}, 2 \mu \mathrm{M} \mathrm{DTT}$, and protease inhibitor mixture. Cells then were spun for 20-30 min at $20,000 \times g$, and the supernatants were used. Antibodies for FAK were added at a dilution of 1:100 to a total of $1 \mathrm{ml}$ and immunoprecipitations were performed at $4^{\circ} \mathrm{C}$ overnight with constant mixing. Fifty to $100 \mu \mathrm{l}$ of protein $\mathrm{A}$ or $\mathrm{G}$ beads were added, and the solution was allowed to mix for $1-3 \mathrm{~h}$ at $4^{\circ} \mathrm{C}$. Immunoprecipitations were washed using the immunoprecipitation buffer $4 \mathrm{x}$ and then brought up in 2x Laemli buffer for protein electrophoresis and Western blot analysis. Supernatants were loaded at one-fiftieth the volume of the pellet.

\section{Establishment and treatment of orthotopic intracranial tumors in athymic nude mice}

Orthotopic tumors were established through intracranial injection of tumor cells into brains of athymic nude mice. Six animals were used for each group. IOMM-Lee cells were grown in 
serum-supplemented DMEM media for two days and trypsinized. 0.1 million cells were injected into anesthetized nude mice (50 mg/kg ketamine, $10 \mathrm{mg} / \mathrm{kg}$ xylazine) using a stereotactic frame.

The animals were maintained for one week to allow the tumors to develop and then divided into 8 treatment groups with 6 animals in each group. Alzet mini-osmotic pumps (Durect Corporation, Cupertino, CA) containing $150 \mu \mathrm{g}$ of plasmid at a concentration of $1.5 \mu \mathrm{g} / \mu \mathrm{L}$ were set up for each animal. The animals were treated with pSV, pUC, pU2 and FLuPAR/ cathepsin $\mathrm{B}$ with or without irradiation and allowed to grow for 3 to 4 weeks. Once the control group showed chronic symptoms, the animals were euthanized by cardiac perfusion - first using PBS followed by $10 \%$ phosphate-buffered formalin. The brains were removed and stored in 10\% phosphate-buffered formalin and embedded in paraffin as per standard protocol [39].

Densitometry. Densitometry was performed by using Image J Software (NIH) to quantify the band intensities obtained from western blot and RT-PCR. Data represent values of three separate experiments.

Statistical analysis. All data here are presented as means \pm $\mathrm{SD}$ of at least three independent experiments, each performed in triplicate. One-way analysis of variance (ANOVA) combined with the Tukey post hoc test of means were used for multiple comparisons in cell culture experiments. Differences in the values were considered significant at $p<0.05$.

\section{References}

1. Louis DN, Scheithauer BW, Budka H, von Deimling A, Kepes JJ (2000) Meningiomas pathology and genetics of tumours of the nervous system. In Kleihues P, Cavenee WK, eds. World health organization classification of tumours. Lyon: IARC Press. pp 176-184.

2. Bouterfa H, Darlapp AR, Klein E, Pietsch T, Roosen K, et al. (1999) Expression of different extracellular matrix components in human brain tumor and melanoma cells in respect to variant culture conditions. J Neurooncol 44: 23-33.

3. Nakada M, Okada Y, Yamashita J (2003) The role of matrix metalloproteinases in glioma invasion. Front Biosci 8: e261-e269.

4. Gondi CS, Kandhukuri N, Dinh DH, Gujrati M, Rao JS (2007) Downregulation of $\mathrm{UPAR}$ and $\mathrm{UPA}$ activates caspase-mediated apoptosis and inhibits the PI3K/AKT pathway. Int J Oncol 31: 19-27.

5. Lakka SS, Gondi CS, Yanamandra N, Olivero WC, Dinh DH, et al. (2004) Inhibition of cathepsin B and MMP-9 gene expression in glioblastoma cell line via RNA interference reduces tumor cell invasion, tumor growth and angiogenesis. Oncogene 23: 4681-4689.

6. Blasi F, Carmeliet P (2002) uPAR: a versatile signalling orchestrator. Nat Rev Mol Cell Biol 3: 932-943.

7. Gondi CS, Lakka SS, Dinh DH, Olivero WC, Gujrati M, et al. (2004) RNAimediated inhibition of cathepsin B and UPAR leads to decreased cell invasion, angiogenesis and tumor growth in gliomas. Oncogene 23: 8486-8496.

8. Jo M, Thomas KS, O'Donnell DM, Gonias SL (2003) Epidermal growth factor receptor-dependent and -independent cell-signaling pathways originating from the urokinase receptor. J Biol Chem 278: 1642-1646.

9. Ossowski L, Aguirre-Ghiso JA (2000) Urokinase receptor and integrin partnership: coordination of signaling for cell adhesion, migration and growth. Curr Opin Cell Biol 12: 613-620.

10. Parsons JT (2003) Focal adhesion kinase: the first ten years. J Cell Sci 116: 1409-1416.

11. Hanks SK, Ryzhova L, Shin NY, Brabek J (2003) Focal adhesion kinase signaling activities and their implications in the control of cell survival and motility. Front Biosci 8: 982-996.

12. Schlaepfer DD, Mitra SK (2004) Multiple connections link FAK to cell motility and invasion. Curr Opin Genet Dev 14: 92-101.

13. Gabarra-Niecko V, Schaller MD, Dunty JM (2003) FAK regulates biological processes important for the pathogenesis of cancer. Cancer Metastasis Rev 22: 359-374.

14. McLean GW, Carragher NO, Avizienyte E, Evans J, Brunton VG, et al. (2005) The role of focal-adhesion kinase in cancer - a new therapeutic opportunity. Nat Rev Cancer 5: 505-515.

15. Lightfoot Jr. HM, Lark A, Livasy CA, Moore DT, Cowan D, et al. (2004) Upregulation of focal adhesion kinase (FAK) expression in ductal carcinoma in situ (DGIS) is an early event in breast tumorigenesis. Breast Cancer Res Treat 88: $109-116$.

16. Oktay MH, Oktay K, Hamele-Bena D, Buyuk A, Koss LG (2003) Focal adhesion kinase as a marker of malignant phenotype in breast and cervical carcinomas. Hum Pathol 34: 240-245.

\section{Supporting Information}

Figure S1 Simultaneous downregulation of uPA, UPAR and cathepsin-B reduce expression of p-FAK (Y925), inhibits cellular proliferation and over expression of uPAR and cathepsin $B$ rescues cellular proliferation in meningiomas.

(TIF)

\section{Acknowledgments}

We thank Shellee Abraham for manuscript preparation, and Diana Meister and Sushma Jasti for manuscript review.

The contents are solely the responsibility of the authors and do not necessarily represent the official views of the National Institutes of Health (NIH).

\section{Author Contributions}

Conceived and designed the experiments: RG JSR. Performed the experiments: RG AKN VRG CG PB. Analyzed the data: RG JDK AJT SM JSR. Contributed reagents/materials/analysis tools: JSR. Wrote the paper: RG. Provided discussion and revision of critically important intellectual content: JSR.

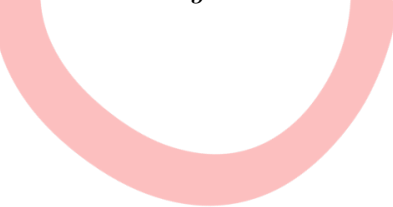

17. Mitra SK, Hanson DA, Schlaepfer DD (2005) Focal adhesion kinase: in command and control of cell motility. Nat Rev Mol Cell Biol 6: 56-68.

18. Barberis L, Wary KK, Fiucci G, Liu F, Hirsch E, et al. (2000) Distinct roles of the adaptor protein She and focal adhesion kinase in integrin signaling to ERK. J Biol Chem 275: 36532-36540.

19. Schlaepfer DD, Hunter T (1997) Focal adhesion kinase overexpression enhances ras-dependent integrin signaling to ERK2/mitogen-activated protein kinase through interactions with and activation of c-Src. J Biol Chem 272: 13189-13195.

20. Schlaepfer DD, Jones KC, Hunter T (1998) Multiple Grb2-mediated integrinstimulated signaling pathways to ERK2/mitogen-activated protein kinase: summation of both c-Src- and focal adhesion kinase-initiated tyrosine phosphorylation events. Mol Cell Biol 18: 2571-2585.

21. Slack-Davis JK, Eblen ST, Zecevic M, Boerner SA, Tarcsafalvi A, et al. (2003) PAK1 phosphorylation of MEK1 regulates fibronectin-stimulated MAPK activation. J Cell Biol 162: 281-291.

22. Cheng JC, Chou CH, Kuo ML, Hsieh CY (2006) Radiation-enhanced hepatocellular carcinoma cell invasion with MMP-9 expression through PI3K/Akt/NF-kappaB signal transduction pathway. Oncogene 25: 7009-7018.

23. Speake WJ, Dean RA, Kumar A, Morris TM, Scholefield JH, et al. (2005) Radiation induced MMP expression from rectal cancer is short lived but contributes to in vitro invasion. Eur J Surg Oncol 31: 869-874.

24. Kunigal S, Lakka SS, Joseph P, Estes N, Rao JS (2008) Matrix metalloproteinase-9 Inhibition Down-Regulates Radiation-Induced Nuclear Factor-\{kappa\}B Activity Leading to Apoptosis in Breast Tumors. Clin Cancer Res 14: 3617-3626.

25. Rao NK, Shi GP, Chapman HA (1995) Urokinase receptor is a multifunctional protein: influence of receptor occupancy on macrophage gene expression. J Clin Invest 96: 465-474.

26. Schmitt M, Janicke F, Moniwa N, Chucholowski N, Pache L, et al. (1992) Tumor-associated urokinase-type plasminogen activator: biological and clinical significance. Biol Chem Hoppe Seyler 373: 611-622.

27. Lee JS, Kim HS, Jung JJ, Kim YB, Park CS, et al. (2001) Correlation between angiogenesis, apoptosis and cell proliferation in invasive ductal carcinoma of the breast and their relation to tumor behavior. Anal Quant Cytol Histol 23: 161-168.

28. Kerr JF, Wyllie AH, Currie AR (1972) Apoptosis: a basic biological phenomenon with wide-ranging implications in tissue kinetics. Br $\mathrm{J}$ Cancer 26: 239-257.

29. Vassalli JD, Sappino AP, Belin D (1991) The plasminogen activator/plasmin system. J Clin Invest 88: 1067-1072.

30. McLean GW, Komiyama NH, Serrels B, Asano H, Reynolds L, et al. (2004) Specific deletion of focal adhesion kinase suppresses tumor formation and blocks malignant progression. Genes Dev 18: 2998-3003.

31. Shen TL, Park AY, Alcaraz A, Peng X, Jang I, et al. (2005) Conditional knockout of focal adhesion kinase in endothelial cells reveals its role in 
angiogenesis and vascular development in late embryogenesis. J Cell Biol 169: 941-952.

32. Hecker TP, Gladson CL (2003) Focal adhesion kinase in cancer. Front Biosci 8: s705-s714.

33. Mitra SK, Schlaepfer DD (2006) Integrin-regulated FAK-Src signaling in normal and cancer cells. Curr Opin Cell Biol 18: 516-523.

34. Miyazaki T, Kato H, Nakajima M, Sohda M, Fukai Y, et al. (2003) FAK overexpression is correlated with tumour invasiveness and lymph node metastasis in oesophageal squamous cell carcinoma. Br J Cancer 89: 140145.

35. Itoh S, Maeda T, Shimada M, Aishima S, Shirabe K, et al. (2004) Role of expression of focal adhesion kinase in progression of hepatocellular carcinoma. Clin Cancer Res 10: 2812-2817.
36. Recher C, Ysebaert L, Beyne-Rauzy O, Mansat-De MV, Ruidavets JB, et al. (2004) Expression of focal adhesion kinase in acute myeloid leukemia is associated with enhanced blast migration, increased cellularity, and poor prognosis. Cancer Res 64: 3191-3197.

37. Sood AK, Coffin JE, Schneider GB, Fletcher MS, DeYoung BR, et al. (2004) Biological significance of focal adhesion kinase in ovarian cancer: role in migration and invasion. Am J Pathol 165: 1087-1095.

38. Leunig M, Yuan F, Menger MD, Boucher Y, Goetz AE, et al. (1992) Angiogenesis, microvascular architecture, microhemodynamics, and interstitial fluid pressure during early growth of human adenocarcinoma LS174T in SCID mice. Cancer Res 52: 6553-6560.

39. Graham FL, Prevec L (1991) Manipulation of adenovirus vectors; in Murray EJ, ed. Methods in Molecular Biology. CliftonNJ: The Humana Press. pp 109-127.
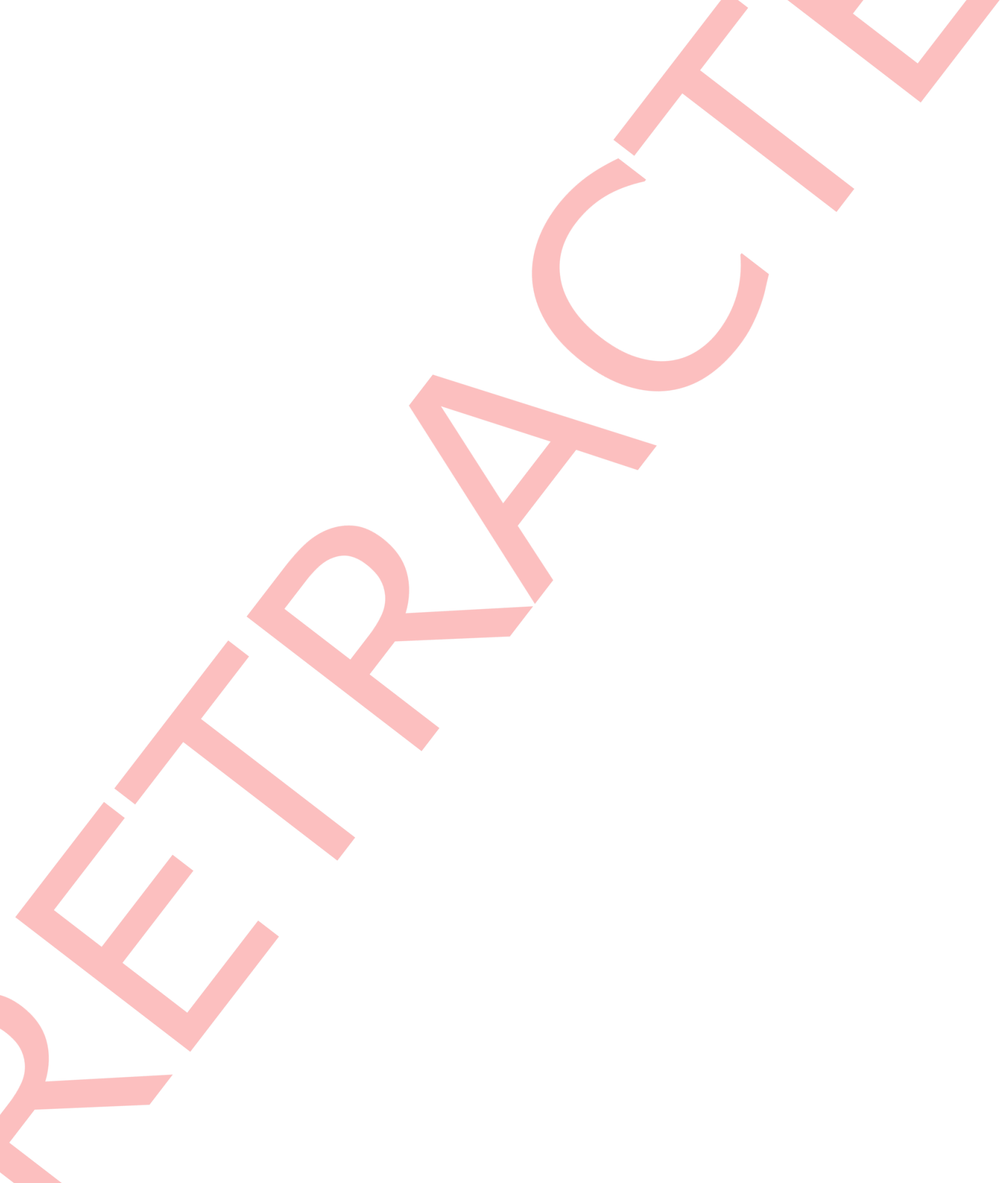